\title{
New development of Immune checkpoints blockade in cancer immunotherapy
}

\author{
Wu Feixuan ${ }^{1, a}$ \\ unit1,building15, Zhong heqiao Road30, Qinhuai District, Nanjing, Jiangsu Province, China
}

\begin{abstract}
Immunotherapy has become the main stream in cancer treatment nowadays. It includes $\mathrm{T}$ cell, NK cell targeted therapy, as well as antibody targeted therapy and its derivatives. Recently immune checkpoints blockade (ICB) has been developed, which are said to be a better method in treatment. The release of negative regulators of immune activation has resulted in unprecedented rates of long-lasting tumor responses in patients with a variety of cancers. This can be achieved by antibodies blocking the cytotoxic $\mathrm{T}$ lymphocyte-associated protein 4 (CTLA-4), the programmed cell death 1 (PD-1) and programmed cell death ligand 1 (PDL-1) pathway or the lymphocyte-activated gene-3 (LAG-3) pathway, either alone or in combination. Improvement of treatment benefits from the research in molecular mechanisms of ICB. For example, mechanism of LAG-3 and its valid ligands is unclear, which leads to a misunderstanding that the antibody might be ineffective. After finding these results demonstrating that fibrinogen-like protein 1(FGL1) is an important functional ligand of LAG-3, it reveals the role of this LAG 3-FGL1 pathway in tumor immunity. Although there are some potential side effects, these therapies turn out to have lots of positive effects on most patients. Therefore, this review summarizes the latest advances, hoping that it may have a great contribution to the cancer treatment.
\end{abstract}

\section{Introduction of Immune checkpoints}

There are several types of immune checkpoints based on what they target at. The majority of immune checkpoints are expressed on T cells such as PD-1, PDL-1, CD137, BTLA, CTLA-4, B7-1/2 and CD40 while PD-1, HVEM, CD160, CD226 and TIGIT can also be expressed on natural killer cells. Signal-regulatory protein (SIRP)a, LILRB1, and sialic-acid-binding Ig-like lectin 10 (Siglec10) are inhibitory receptors expressed on myeloid cells, including macrophages, dendritic cells (DCs) and neutrophils $[1,2]$.

\section{PD-1 and PDL-1}

In 1992, Honjo Tasuku first identified PD-1 as an induction gene on activated $\mathrm{T}$ lymphocytes, which made a significant contribution to the establishment of the principle of cancer immunotherapy by blocking PD-1. Then, in 1999, Chen Lieping reported PDL-1, the third member of the B7 family, and found that B7-H1 was different from the previously reported members of the B7 family in that it could not bind CD28, CTLA-4 and ICOS, but could promote the secretion of IL-10.

PD-1, a member of the CD28 family, acts as a negative regulator of immune response preferentially in peripheral tissues through the interaction with PDL-1 or PDL-2. Part of a negative feedback loop after immune activation, PD- 1 and PDL- $1 / 2$ plays a role in the maintenance of peripheral tolerance, leting down following immune activation and chronic infections. PD1 is expressed on lymphocytes, monocytes, natural killer [NK) cells, and dendritic cells due to a part of peripheral immune suppression[3]. Importantly, PD-1 is absent on resting or naïve $\mathrm{T}$ cells and is transiently upregulated during the activation process. This upregulation is analogous to the development of $\mathrm{T}$ cells in the thymus, with increased PD-1 which is required for positive and negative selection of immature $\mathrm{T}$ cells following TCR activation[4]. Upon activation $\mathrm{T}$ cells upregulate the transcription of PDCD1, the gene locus for PD-1. The transcriptional regulation of PD-1 is different from CTLA-4, which localizes to the plasma membrane quickly after TCR signaling[5]. PD-L1 is broadly expressed, and the protein is easy to be induced by many cytokines, particularly type 1 and type 2 interferons [6-9].

The first evidence of the antitumor activity of PD-1 blockade was with the fully human monoclonal antibody nivolumab. It was first administered to a patient in October 2006 in a phase 1 single infusion dose-escalation trial. Then, the anti-PD-1 antibody pembrolizumab entered clinical testing in April 2011. Antitumor activity of PD-1-pathway blockade has been observed in a subset of patients within a broad range of cancers, particularly in carcinogen-induced cancers or cancers driven by viral infections. There are the highest antitumor activities of single-agent PD-1-blockade therapy in Hodgkin's lymphoma, with constitutive expression of PD-L1 through a common amplification of the PD-L1-encoding locus together with PD-L2 and Janus kinase 2 (JAK2).

\footnotetext{
${ }^{a}$ Corresponding author: Feixuan_wu@163.com
} 
There are other two approvals of single agent anti-PD-1 therapies in hepatocellular carcinoma, with its known relationship to hepatitis virus infection[10], and renal cell carcinoma[11], which has a low single-nucleotide mutational load but a higher frequency of indels than other common cancers, leading to increased immunogenicity[12]. The first anti-PD-L1 antibody approved was atezolizumab for urothelial cancers in 2016 , followed by avelumab for Merkel cell carcinoma in 2017. Besides, pembrolizumab's clinical development focused on patients with metastatic melanoma and NSCLC because of the encouraging clinical data from nivolumab, resulting in the largest phase 1 trial ever conducted in oncology, eventually enrolling 1235 patients[13, 14]. Now, there is a phase II trial to evaluate the efficacy and safety of SHR-1210 plus apatinib mesylate versus Pemetrexed and Carboplatin in Subjects with KRAS mutant stage IV non-squamous NSCLC.

\section{CTLA-4}

CTLA-4 was demonstrated to have a potent inhibitory role in regulating $\mathrm{T}$ cell responses by two groups, one led by James Allison and the other by Jeffrey Bluestone[1517].

CTLA-4 is a member of the CD28 family and is expressed exclusively by $\mathrm{T}$ lymphocytes. Upon T-cell activation, CTLA-4 within intracellular granules is translocated to the plasma membrane[18], which is fast and allows for CTLA-4-mediated regulation of the amplitude of T-cell responses by regulating T-cell activation and priming. CTLA-4 binds to CD80 or CD86 costimulatory molecules and acts as a competitive antagonist with CD28[19]. In addition, CTLA-4 interacts with protein tyrosine phosphatase 11 and 6 [also known as SHP-1/2) to dampen TCR signal[19]. CTLA-4 also plays a role in one arm of peripheral tolerance, an immunological process to prevent self-reactive immune responses, by dampening $\mathrm{T}$ effector cell function and increasing immunosuppressive Treg activity[20]. Unlike effector cells, Tregs express CTLA-4 constitutively and act as a major mechanism of suppression by Tregs[21]. CTLA-4 on Tregs can compete with CD28 on effector T cells for binding with $\mathrm{CD} 80 / 86$ on APCs, thus suppressing T-cell activation. The higher level of CTLA4 on Tregs also serves to preferentially deplete Tregs in tumors treated with anti-CTLA-4 therapies[22].

At first, two fully human CTLA-4-blocking antibodies, ipilimumab and tremelimumab, entered clinical trials in patients with advanced cancer in 2000 . Although these were relatively infrequent and accompanied by a set of mechanism-related toxicities resulting from tissue-specific inflammation, it became apparent that durable tumor regressions could occur quickly $[23,24]$. The most apparent clinical activity of CTLA-4 blockade was in patients with advanced metastatic melanoma, with a $15 \%$ rate of objective radiographic response that has been durable in some patients for $>10$ years since stopping therapy. Additional CTLA-4-blocking antibodies have recently entered clinical trials (NCT02694822). Abatacept is a biologically engineered CTLA-4-mimetic which is approved as an intravenous (IV) infusion to treat adult psoriatic arthritis, adult rheumatoid arthritis, and juvenile idiopathic arthritis because abatacept mimics CTLA-4 function.

\section{LAG-3}

In 1990, Frédéric Triebel first discovered LAG-3, a surface molecule highly homologous to CD4 in structure, but with less than $20 \%$ identity at the amino acid level[25]. LAG-3 binds to MHC class II molecules like CD4, but with a much higher affinity[26]. LAG-3 is expressed on activated CD4 and CD8 T cells, and on activated Tregs [27] and $\operatorname{Tr} 1$ cells[28, 29]. It is also expressed on a subset of NK cells [30], B cells[31] and plasmacytoid DCs [32]. LAG-3 is localized and degraded within the lysosomal compartments in resting T cells [33, 34]. After stimulation, LAG-3 can be rapidly translocated to the cell surface where its expression is regulated by two TCR-induced metalloproteases (ADAM10 and ADAM17). LAG-3 cleavage from the cell surface by these metalloproteases allows for normal T-cell activation[35]. It finally turns out that LAG-3 is only transiently expressed at the surface of activated T cells which are stimulated in acute conditions, even if it remains high on $\mathrm{T}$ cells stimulated within tolerizing environments[26, 36].

However, the major ligand that mediates the immune suppressive functions of LAG-3 remains controversial. Initial studies by Baixeras et al [30] showed an interaction between MHC-II and LAG-3 via a cell-cell adhesion assay, which was further extended by studies indicating LAG-3 fusion protein binding to $\mathrm{MHC}-\mathrm{II}^{+} \mathrm{B}$ cell lines[37, 38]. But at the same time, there was a lack of direct evidence for the protein-protein interaction between LAG-3 and MHC-II. Recently, Wang et al found that fibrinogen-like protein 1 (FGL1), a liver-secreted protein, was a major LAG-3 functional ligand independent from MHC-II. When it is under normal physiological conditions, FGL1 protein is primarily secreted from hepatocytes and contributes to its mitogenic and metabolic functions [39-44]. FGL1 inhibits antigen-specific $\mathrm{T}$ cell activation, and ablation of FGL1 in mice promotes T cell immunity. Blockade of the FGL1-LAG-3 interaction by monoclonal antibodies stimulating tumor immunity is therapeutically against established mouse tumors in a receptor-ligand interdependent manner. Elevated FGL1 in the plasma of cancer patients is associated with a poor prognosis and resistance to anti-PD-1/B7-H1 therapy.

Relatlimab, also known as bms-986016, is a monoclonal antibody targeting LAG-3. In the clinical phase 1/2a trial called ca224-020, relatlimab and the PD1 inhibitor Opdivo constitute a combination therapy to treat solid tumor patients, including melanoma patients who do not respond to or develop resistance to PD-1 / PDL-1 immunotherapy. Additionally, based on the latest result, tumor immune cells express LAG - around 3 for relatlimab and Opdivo combination therapy in patients with objective response rate (ORR) is $18 \%$, the 
expression of LAG-3 tumor immune cells around ORR of $5 \%$ less than $1 \%$ of the patients the results confirm the LAG-3 is a valid target.[45].

\section{Improvement of immune checkpoint therapies}

The remarkable effect of immune checkpoint therapies has been widely proved in terms of a range of tumor treatment. However, it is inevitable to lead to some undesirable effects, such as fatigue, diarrhea, rash and so on[7]. In order to gain a better effect and reduce pain, there have been some improved treatment options developed, most of which exert effect through combinations. Combination CTLA-4 and PD-1-blockade therapy had a slightly higher 3-year survival than patients initially receiving nivolumab alone (58\% versus $52 \%$ ), yet with higher frequency of toxicity $[23,27]$. ICB is also being investigated in combination with a wide range of agents, bispecific $\mathrm{T}$ cell engager[26], hypomethylating agents [46], cytokine[47] and chimeric antigen receptor $\mathrm{T}$ (CAR-T)[21], and other traditional way especially radiotherapy and chemotherapy[48].

\section{Conclusion}

So far, there have been many immune checkpoints discovered and utilized in cancer therapies. PD-1, PDL-1, CTLA-4 and LAG-3 are the most effective checkpoints. The mechanisms of former three seem to be clearer than the last one which is still under development. Building on recent success in this field is important, but continuing to incorporate the emerging knowledge from mechanistic basic-science studies is critical to achieve greater therapeutic success. On one hand, more and more efficient combined therapies, CTLA-4 and PD-1blockade, and ICB with cytokine, have been discovered and used to reduce side effects and increase the therapeutic effect. For another, the mechanism of LAG-3 is still under investigation, indicating great potential within it. Therefore, it is expected that more ligands like LAG-3 would be discovered in the future, leading to broader scope of knowledge and more efficient treatments to help treat different types of cancer.

\section{Reference}

1. T. Matozaki, Y. O. Murata, Hideki, H. Ohnish, Functions and molecular mechanisms of the CD47SIRP alpha signalling pathway. Trends in Cell Biology 19, 72-80 (2009).

2. A. A. Barkal et al., Engagement of MHC class I by the inhibitory receptor LILRB1 suppresses macrophages and is a target of cancer immunotherapy. Nature Immunology 19, 76-84 (2018)

3. M. E. Keir, G. J. Butte MJFreeman, PD-1 and its ligands in tolerance and immunity. Annual Review of Immunology 26, 677-704 (2008).

4. C. Blank et al., Absence of Programmed Death
Receptor 1 Alters Thymic Development and Enhances Generation of CD4/CD8 Double-Negative TCR-Transgenic T Cells. The Journal of Immunology 171, 4574-4581 (2003).

5. P. S. Linsley et al., Intracellular Trafficking of CTLA-4 and Focal Localization Towards Sites of TCR Engagement. Immunity 4, 535 (1996).

6. H. Dong et al., Tumor-associated B7-H1 promotes Tcell apoptosis: a potential mechanism of immune evasion. Nature Medicine 8, 793-800 (2002).

7. Expression of the B7 - related molecule B7 - H1 by glioma cells: a potential mechanism of immune paralysis. Chinese Journal of Neurooncology, (2003).

8. A. Garcia-Diaz et al., Interferon Receptor Signaling Pathways Regulating PD-L1 and PD-L2 Expression. Cell Reports 19, 1189 (2017).

9. N. Borcherding et al., Keeping tumors in check: A mechanistic review of clinical response and resistance to immune checkpoint blockade in cancer. Journal of Molecular Biology, (2018).

10. B. Schreiner et al., Interferon- $\beta$ enhances monocyte and dendritic cell expression of B7-H1 (PD-L1), a strong inhibitor of autologous T-cell activation: relevance for the immune modulatory effect in multiple sclerosis. Journal of Neuroimmunology $\mathbf{1 5 5}$, 172-182 (2004).

11. J. M. Chemnitz, R. V. Parry, K. E. Nichols, C. H. June, J. L. Riley, SHP-1 and SHP-2 associate with immunoreceptor tyrosine-based switch motif of programmed death 1 upon primary human $\mathrm{T}$ cell stimulation, but only receptor ligation prevents $\mathrm{T}$ cell activation. Journal of Immunology 173, 945-954 (2004).

12. K. A. Sheppard et al., PD-1 inhibits T-cell receptor induced phosphorylation of the ZAP70/CD3 $\zeta$ signalosome and downstream signaling to $\mathrm{PKC} \theta$. Febs Letters 574, 37-41 (2004).

13. E. B. Garon et al., Pembrolizumab for the treatment of non-small-cell lung cancer. New England Journal of Medicine 372, 2018 (2015)

14. A. Ribas et al., Association of Pembrolizumab With Tumor Response and Survival Among Patients With Advanced Melanoma. Journal of the American Medical Association 315, 1600 (2016).

15. C. A. Chambers, M. S. Kuhns, J. G. Egen, J. P. Allison, CTLA-4-mediated inhibition in regulation of $\mathrm{T}$ cell responses: mechanisms and manipulation in tumor immunotherapy. Annual Review of Immunology 19, 565-594 (2000).

16. D. R. Leach, M. F. Krummel, J. P. Allison, Enhancement of antitumor immunity by CTLA-4 blockade. Science 271, 1734-1736 (1996).

17. T. L. Walunas et al., CTLA-4 can function as a negative regulator of $\mathrm{T}$ cell activation. Immunity $\mathbf{1}$, 405-413 (1994).

18. D. M. Pardoll, The blockade of immune checkpoints in cancer immunotherapy. Nature Reviews Cancer 12, 252-264 (2012).

19. A. Ribas, Adaptive Immune Resistance: How Cancer Protects from Immune Attack. Cancer Discovery 5, 915 (2015). 
20. A. Garciadiaz et al., Interferon Receptor Signaling Pathways Regulating PD-L1 and PD-L2 Expression. Cell Reports 19, 1189 (2017).

21. K. E. Pauken et al., Epigenetic stability of exhausted $\mathrm{T}$ cells limits durability of reinvigoration by PD-1 blockade. Science 354, 1160-1165 (2016).

22. D. S. Shin et al., Primary Resistance to PD-1 Blockade Mediated by JAK1/2 Mutations. Cancer Discovery 7, 188 (2016).

23. H. F Stephen et al., Biologic activity of cytotoxic T lymphocyte-associated antigen 4 antibody blockade in previously vaccinated metastatic melanoma and ovarian carcinoma patients. Proc Natl Acad Sci US A 100, 4712-4717 (2003).

24. A. Ribas et al., Phase 1 trial of monthly doses of the human anti-CTLA4 monoclonal antibody CP675,206 in patients with advanced melanoma. Journal of Clinical Oncology 23, 7524-7524 (2005).

25. F. Triebel, . et al., LAG-3, a novel lymphocyte activation gene closely related to CD4. Journal of Experimental Medicine 171, 1393-1405 (1990).

26. B. Huard, ., P. Prigent, ., M. Tournier, ., D. Bruniquel, ., F. Triebel, . CD4/major histocompatibility complex class II interaction analyzed with CD4- and lymphocyte activation gene3 (LAG-3)-Ig fusion proteins. European Journal of Immunology 25, 2718-2721 (2010).

27. C. T. Huang et al., Role of LAG-3 in Regulatory T Cells. Immunity 21, 503-513 (2004).

28. G. Nicola et al., Coexpression of CD49b and LAG-3 identifies human and mouse $\mathrm{T}$ regulatory type 1 cells. Nature Medicine 19, 739-746 (2013).

29. O. Tomohisa et al., CD4+CD25-LAG3+ regulatory $\mathrm{T}$ cells controlled by the transcription factor Egr-2. Proceedings of the National Academy of Sciences of the United States of America 106, 13974-13979 (2009).

30. E. Baixeras et al., Characterization of the lymphocyte activation gene 3 -encoded protein. A new ligand for human leukocyte antigen class II antigens. Journal of Experimental Medicine 176, 327-337 (1992).

31. K. Malgorzata, K. Jan, C. S. Giuseppina, K. Klaus, Expression of lymphocyte activation gene 3 (LAG-3) on B cells is induced by T cells. European Journal of Immunology 35, 2081-2088 (2005).

32. C. J. Workman et al., LAG-3 regulates plasmacytoid dendritic cell homeostasis. Journal of immunology (Baltimore, Md. : 1950) 182, 1885-1891 (2009).

33. B. Joonbeom, L. Suk Jun, P. Chung-Gyu, L. Young Sik, C. Taehoon, Trafficking of LAG-3 to the surface on activated $\mathrm{T}$ cells via its cytoplasmic domain and protein kinase C signaling. Journal of Immunology 193, 3101-3112 (2014).

34. S. R. Woo et al., Differential subcellular localization of the regulatory T-cell protein LAG-3 and the coreceptor CD4. European Journal of Immunology 40, 1768-1777 (2010).

35. L. Nianyu et al., Metalloproteases regulate T-cell proliferation and effector function via LAG-3. Embo Journal 26, 494-504 (2014).

36. J. F. Grosso et al., Functionally distinct LAG-3 and
PD-1 subsets on activated and chronically stimulated CD8 T cells. Journal of Immunology 182, 6659-6669 (2009).

37. B. Huard, P. Prigent, F. Pages, D. Bruniquel, F. Triebel, $\mathrm{T}$ cell major histocompatibility complex class II molecules down-regulate CD4+ T cell clone responses following LAG-3 binding. Eur J Immunol 26, 1180-1186 (1996).

38. B. Huard, P. Prigent, M. Tournier, D. Bruniquel, F. Triebel, CD4/major histocompatibility complex class II interaction analyzed with CD4- and lymphocyte activation gene-3 (LAG-3)-Ig fusion proteins. Eur $J$ Immunol 25, 2718-2721 (1995).

39. V. Demchev et al., Targeted deletion of fibrinogen like protein 1 reveals a novel role in energy substrate utilization. Plos One 8, e58084 (2013).

40. H. Hara et al., Molecular cloning and functional expression analysis of a cDNA for human hepassocin, a liver-specific protein with hepatocyte mitogenic activity. BBA - Gene Structure and Expression 1520, 45-53 (2001).

41. L. Chang-Yan et al., Recombinant human hepassocin stimulates proliferation of hepatocytes in vivo and improves survival in rats with fulminant hepatic failure. Gut 59, 817 (2010)

42. Z. Liu, C. Ukomadu, Fibrinogen-like-protein 1, a hepatocyte derived protein is an acute phase reactant. Biochemical \& Biophysical Research Communications 365, 729-734 (2008).

43. T. Yamamoto et al., Molecular Cloning and Initial Characterization of a Novel Fibrinogen-Related Gene, HFREP-1. Biochemical \& Biophysical Research Communications 193, 681-687 (1993).

44. J. Yan et al., Cloning and characterization of a mouse liver-specific gene mfrep-1, up-regulated in liver regeneration. Cell research 12, 353-361 (2002).

45. I. Le Mercier, J. L. Lines, R. J. Noelle, Beyond CTLA-4 and PD-1, the Generation Z of Negative Checkpoint Regulators. Frontiers in Immunology 6 , (2015).

46. C. Krupka et al., Blockade of the PD-1/PD-L1 axis augments lysis of AML cells by the CD33/CD3 BiTE antibody construct AMG 330: reversing a T-cellinduced immune escape mechanism. Leukemia 30, 484-491 (2016).

47. A. Ribas, J. D. Wolchok, Cancer immunotherapy using checkpoint blockade. Science 359, 1350-1355 (2018).

48. H. Wang et al., Immune checkpoint blockade and CAR-T cell therapy in hematologic malignancies. Journal of hematology \& oncology 12, 59 (2019). 